\title{
A multifaceted, home based, environmental intervention reduced asthma related morbidity in children with atopic asthma
}

Morgan WJ, Crain EF, Gruchalla RS, et al; for the Inner-City Asthma Study Group. Results of a home-based environmental intervention among urban children with asthma. N Engl J Med 2004 9;351:1068-80.

Is a multifaceted, home based, environmental intervention (HBEI) effective for improving asthma related outcomes in inner city children with atopic asthma?

\section{METHODS}

Design: randomised controlled trial.

Allocation: $\{$ concealed\}.*

Blinding: blinded (outcome assessors).

Follow up period: 1 year of intervention followed up by a 1 year observational period.

Setting: 7 major cities in the US

Patients: 937 children $5-11$ years of age (mean age $7.7 y$, 63\% boys) who had atopic asthma and resided in districts in which $\geqslant 20 \%$ of households had incomes below the federal poverty level. Children were also required to have had $\geqslant 1$ asthma related hospital admission or 2 unscheduled asthma related visits to a clinic or emergency department during the previous 6 months and a positive skin test in response to $\geqslant 1$ of 11 indoor allergens.

$\mathbf{R}_{\mathbf{X}}$

Intervention: a 1 year HBEl $(n=469)$ or home evaluation only (control group, $n=468$ ). The HBEl was tailored to each child's sensitisation and environmental risk profile. Each child's caretaker was provided with education and resources (eg, allergy control mattress covers, high efficiency vacuum cleaners, and air purifiers) to reduce exposure to both allergens and tobacco smoke. The HBEI was organised in 6 modules that focused on reducing exposure to dust mites, passive smoking, cockroaches, pets, rodents, and mould. All families received visits for home evaluation (including measurement of allergen concentrations) at baseline and at 6 month intervals throughout the study.

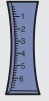

Outcomes: maximal number of days with symptoms (defined as the largest value among 3 variables: number of days with wheezing, tightness in the chest, or cough; number of nights with disturbed sleep as a result of asthma; and number of days on which the child had to slow down or discontinue play activities because of asthma) in the 2 weeks before telephone interviews (done every 2 mo throughout the study).

IILD Patient follow up: $93 \%$ at 1 year and $88 \%$ at 2 years (intention to treat analysis).

*Information provided by author.

\section{MAIN RESULTS}

The mean maximal number of days with symptoms per each 2 week period was lower in the HBEI group than in the control group during For correspondence: Dr W J Morgan, Arizona Respiratory Center, University of Arizona, Tucson, AZ, USA. wmorgan@resp-sci.arizona.edu

Sources of funding: National Institute of Allergy and Infectious Disease; National Institute of Environmental Health Sciences; National Center for Research Resources. the first ( $3.4 v 4.2 \mathrm{~d}, \mathrm{p}<0.001)$ and second years $(2.6 v 3.2, \mathrm{p}<0.001)$. Reduction in allergen concentrations (eg, Dermatophagoides farinae and the cockroach allergen Bla $g l$ in the bedroom) was greater in the HBEI group than in the control group ( $\mathrm{p}$ values $<0.05$ ).

\section{CONCLUSION}

A multifaceted, home based, environmental intervention reduced exposure to indoor allergens and asthma related morbidity in inner city children with atopic asthma.

A modified version of this abstract appears in Evidence-Based Medicine

Commentary

he multisite trial by Morgan et al showed that a complex intervention (HBEI) was effective for families of children with asthma. The finding that reduction in allergen exposure correlated with reduced morbidity suggested a direct relation. Interestingly, however, the interventions in this study did not have a significant effect on lung function. Morgan et al reported that the reduction in morbidity could have been a result of the effort to improve the indoor environment as a whole, rather than focusing only on decreasing exposure to one allergen as has been done in previous studies. The complexity of the HBEl in this study, however, renders it impossible to identify the effectiveness of the individual elements. Additionally, because no direct measurement of the children's exposure to environmental tobacco smoke was made, it is unclear whether advice to parents about tobacco smoke exposure had any effect. Previous research supports the conclusion that tobacco smoking by parents increases the risk of asthma related symptoms. However, a meta-analysis of studies that assessed the effect of smoking control programmes on children's exposure to environmental tobacco smoke showed limited effectiveness of brief smoking control programmes. ${ }^{2}$ Furthermore, previous studies have not shown any effect from interventions related to house dust mite control similar to those used in this study. ${ }^{3}$ The study did not determine how much of the effect was a result of education of the parents and how much was a result of physical changes to the child's bedroom (ie, mattress and pillow covers, vacuum, air purifier, pest control). The interventions were quite expensive as reported by the researchers (initial cost of $\$ 1500-\$ 2000$ ). Most, if not all of the interventions would not be covered by any existing North American insurance plans. Although the cost decreases the feasibility of the multifaceted intervention, clinicians might consider advising parents on individualised multiple environmental measures-including smoking cessation - while recognising that it is uncertain how much each separate intervention can reduce asthma symptoms.

Robin Rider Wilkerson, RN, PhD University of Mississippi School of Nursing Madison, Mississippi, USA

1 Cook DG, Strachan DP. Health effects of passive smoking. 3. Parental smoking and prevalence of respiratory symptoms and asthma in school age children. Thorax 1997; 52:1081-94.

2 Roseby R, Waters E, Polnay A, et al. Family and carer smoking control programmes for reducing children's exposure to environmental tobacco smoke. Cochrane Database Syst Rev 2003;(3):CD001746.

3 Gøizsche P, Johansen H, Schmidt L, et al. House dust mite control measures for asthma. Cochrane Database Syst Rev 2004;(4):CD001 187. 